\title{
A Demand - Adaptive MAC Protocol without Information Packet Collision for VANET Safety Services
}

\author{
Qiubo Huang ${ }^{1, a}$ and Zhixiang Zhang ${ }^{1, b^{*}}$ \\ ${ }^{1}$ Donghua University, Shanghai, China

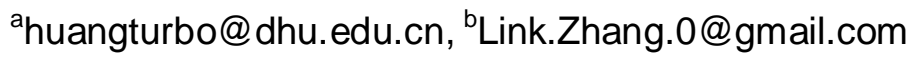 \\ *The corresponding author
}

\section{Keywords: VANET; Safety Services; MAC protocol; Demand-Adaptive}

\begin{abstract}
VANETs, as a particular form of MANETs in which trajectory is restricted by the road and the capacity is not considered, will play the extremely vital role in next generation ITS architecture. Among various services provided by VANET, it's a milestone to reach the guaranteed delivery of urgent safety messages. This paper presents a Demand-Adaptive MAC (DAM) protocol without information packet collision, tailored to security services in vehicular networks and based on CSMA/CA and dynamic TDMA mechanism. In our protocol, Road Side Units (RSUs), the masters in the network, are exploited to response to the application, schedule time slot assignment and broadcast control packets, while vehicles which are the slaves perform under the control. By shrinking or expanding the slots assignment in application and information periods, DAM protocol can realize the self-adaptive demand. By utilizing the principal-subordinate model and a series of architectural mechanisms, the protocol can avoid collision of packets containing the safety information. We simulate the protocol on OPNET platform for a reasonable evaluation and compare it with the modified IEEE 802.11p standard and the ACFM protocol under various traffic scenarios. The simulation results indicate that the DAM protocol outperforms other protocols mentioned above in terms of average end-to-end delay and packet loss rate.
\end{abstract}

\section{Introduction}

In the nearly five years, road traffic safety accidents have constituted a major social issue world-wide. In 2013, road traffic accidents in the Member States of the European Union are claimed more than 1.3 million injuries and about 26 thousand fatalities [1]. In 2014, more than 32 thousand Americans were fatally injured in motor vehicle crashes [2]. In 2015, the number of Chinese fatalities per ten thousand vehicles is 2.6 [3]. Utilizing the statistical data of the car parc, we can roughly calculate the death toll is about 36 thousand. In view of the global vehicle safety problems, the expectation of VANET environment might be quite high. However, Vehicle-to-RSU (V2R) and Vehicle-to-Vehicle $(\mathrm{V} 2 \mathrm{~V})$ communication can potentially reduce the incidence of traffic accidents by delivering safety messages containing specific information (e.g, position, velocity, direction, warning). And it is rough estimated that more than a half of rear-end accidents can be prevented if drivers have the prediction of alert time [4]. Thus, the design of a new reliable wireless MAC protocol to disseminate urgent information with low end-to-end latency and high delivery ratio, by utilizing the advantages of the existing agreements is vital as well as challenging.

VANET, compared to other networks, has its unique features and puzzles. Wireless communications are used in VANET, while wired communications are used in the traditional ones. Due to the existence of "Shadowing Effect" [5] and "Near-far Effect" [6] in wireless transmission, the detection of the conflicts in VANET has some unresolved technical problems such as equipment must be able to send from the time as to receive. And due to the vehicles' high mobility, channels' multi-interference and rapidly changing topologies, the available MAC protocols are very limited. Furthermore, the problems of network storm, hidden terminal and exposed terminal continue to perplex the researchers.

To meet the requirements of real scenarios, people concentrate on well-designed MAC protocols. Two reference standards were presented for $\mathrm{V} 2 \mathrm{~V}$ communications, the North American 
WAVE/DSRC standard [7] and the European ITS-G5 standard [6]. However, both are based on IEEE 802.11p standard which adopts CSMA with collision avoidance(CSMA/CA) and uses seven-segment $10 \mathrm{MHz}$ channels from 5.850 to $5.925 \mathrm{GHz}$. Due to the adoption of CSMA/CA, there are higher chances of collisions and unbounded delay which unbearable for vehicles in case of heavy load conditions [8,9]. And due to the most lack of the optional ACK schedule in 802.11p, it's hard to guarantee QoS for packet delivery and the perception of neighbors. To overcome these difficulties, another channel access method called TDMA can be used into VANET. In TDMA schema, the use of the channel is based on the time slot which could be better.

In this paper, we propose a Demand-Adaptive MAC (DAM) protocol based on dynamic TDMA and CSMA/CA, a novel approach for security services to avoid the collision of information packets considering efficiency and fairness. This protocol takes advantages of TDMA and avoids the disadvantages of CSMA to achieve the goals. And according to our simulation results, the DAM protocol demonstrates a gratifying result in average end-to-end delay and packet loss rate.

\section{Related Work}

Similar to the cable medium in wired network, wireless channel is the critical resource shared by all inter-domain vehicles. When there is an intersection of two vehicles transmitting messages, the collision occurs. So once there is a vehicle sending messages, all the neighbors must wait until the wireless medium is released. What' more, it's hard to determine whether or not a collision occurs for the vehicle which transmitting messages in wireless communication. Thousands of scholars and researchers proposed various access mechanism algorithms and protocols for VANETs to reduce average end-to-end delay and packet loss rate.

The easiest way to control medium access is ALOHA protocol which is unbearable with high collision rate and low reliability [10]. In ALOHA protocol, every terminal uses the simplest method of random access and experimental super high frequency for information dissemination. And there are several fixed allocation protocols with low resource utilization but not suitable for the volatile environment, like: SDMA. The key of SDMA is dividing the road into separated cells which considered in $[11,12]$. It's a waste to assign resources to the no-vehicle cells. And the scheme is not fair to cells with different vehicle densities.

The relatively universal MAC protocol based on probability is Carrier Sense Multiple Access (CSMA), which is adopted by IEEE 802.11p [13]. Kormas, the author of [14], presented a Urban Multi-hop Broadcast (UMB) protocol to handle hidden terminal problem and broadcasting-storm problem. However, the repeaters and black-bursts require more resources. In [15], the Ad-hoc Multi-hop Broadcast (AMB) protocol was proposed as a revised edition of UMB to avoid repeaters by using directional broadcast. Based on CSMA scheme, it requires the back off strategy to randomly choose a time from Content Window (CW). And several optimization methods proposed. MILD algorithm makes the back off time counter 1.5 times when collisions occur and minus one when successful [16]. However, using node's own state to measure the state of network is not good enough. In a new back off algorithm termed Linear/Multiplicative Increase and Linear Decrease (LMILD) [17], only colliding nodes multiplicatively increase, while the overhearing nodes increase linearly. The EIED [18] algorithm uses exponential increase or decrease to enhance the performance of IEEE 802.11p. And a widely used algorithm named HBAB [19] modifies the BEB algorithm to adaptively adjust the $\mathrm{CW}$, which shows up to $50 \%$ in average packet delay compared to the original one.

Another rising approach is using dynamic TDMA, which divides the use of wireless channel into time slides and coordinate access the medium. A novel centralized TDMA [20] avails itself of RSUs to gathering and scheduling based on a new weight-factor-based scheduler, which requires large number of RSUs. Amit Dua et al. [8] proposed a modified TDMA based virtual back off algorithm for adaptive data dissemination. They assume that the arrival of vehicles is subject to Poisson distribution and the service follows the exponential distribution. In spite of the good performance of end-to-end delay, projections are not always reliable under the practical environment, particularly with regard to life and security. Self-organized TDMA (STDMA) was introduced utilizing the 
spatial information (vehicles current location and velocity information) to predict the relative distances. However, broadcasting of cooperative awareness messages (CAM) creates the additional network burden. In [21], the author designed the PTMAC protocol for both two-way traffic and four-way intersections. Potential collisions among vehicles currently out of two-hop communication range can be detected, predicted and eliminated in the protocol. But the protocol is only suitable to four-way traffic. Its suitability and expansibility waits for further enhancing. Weijie Guo presented an adaptive collision-free MAC (ACFM) [22] protocol, in which time cycle consists of $\mathrm{N}$ frames, where $\mathrm{N}$ varies from one to five according to the vehicles' number. But it is a waste to allocate time slot for the vehicles which don't transmit message. And each frame consists of eighteen slots which is not carefully mapped of the varied physical environment. What's more, the expansion of the cycle will definitely cause collisions which seriously affect the normal use of the vehicles that already have gotten the time slots.

\section{Framework of DAM Protocol}

Overview and Assumptions. Our DAM is a cooperative protocol based on CSMA/CA and dynamic TDMA, which is tailored to security services in vehicular networks. The RSU and vehicles in the management domain (DM) could be the independent ecosystem, where the master schedules dynamically round by round. Once vehicle enter into the RSU's DM, it should listen until getting the control message from the master. After resolving the broadcast control message, the vehicle could apply for a limited amount of time slots according to its own need. After the vehicle gets the authorization, it can send message in its next coming time slots. DAM requires RSUs to administrate the applications. When the network traffic gets higher in the particular subnet, the RSU will expand the assignment of time slots and shrink the size of applying in the next round. In contrast, if there are decreasing vehicles needing to send information in the subnet, RSU will shrink the assignment of time slots and expand the size of applying in the next round depending on the policy. Based on the slots policy and authorization, DAM could make the unused time slots remain low and maintain fairness in the complex environment. What' more, the RSU could broadcast to control different algorithms in the apply stages.

We make the following reasonable assumptions in our DAM protocols:

- Adjacent RSUs have no interference in each other by using different frequencies.

-Vehicles know the positions and work frequencies of all the RSUs. And vehicles could communicate to them by adjusting to the same frequency.

-Each vehicle is equipped with dual separate antennas for receiving and sending.

-Each RSU and vehicle must strictly abide by the provisions of DAM protocols, which is vital to accurateness and effectiveness.

- The loss of the packets is only caused by packet collision.

-A unique ID number is given to each vehicle and RSUs.

-All the equipment is in work condition. And there is no device failure.

-When more than one packets arrive at a vehicle or RSU at the same time, all of them will be dropped.

- The time of calculation and processing is negligible owing to the powerful CPU and large data processing capacity.

PHY Layer Model. Since our study is focused on the MAC layer, the physical layer's specific parameters are totally same as the STDMA recommended parameters in [23]. And Doppler phenomenon [9] is neglected. Thus, it's the signal to interference plus noise ratio (SINR) that determines whether the communication is successful or not: 


$$
\operatorname{SINR}\left(\mathrm{V}_{\mathrm{i}}\right)=\frac{\frac{\mathrm{F}_{\mathrm{i}}}{\ell\left(\left|\mathrm{X}_{\mathrm{V}_{\mathrm{i}}}-\mathrm{X}_{\mathrm{V}_{\mathrm{r}}}\right|\right)}}{\sum_{\mathrm{j} \neq \mathrm{i}} \frac{\mathrm{F}_{\mathrm{j}}}{\ell\left(\left|\mathrm{X}_{\mathrm{V}_{\mathrm{j}}}-\mathrm{X}_{\mathrm{V}_{\mathrm{r}}}\right|\right)}+\mathrm{N}_{\text {noise }}}
$$

Where $F_{i}$ is the fading random variables of some distribution of $V_{\mathrm{i}}$, while $\mathrm{X}_{\mathrm{i}}$ is the position of $i$ and $N_{\text {noise }}$ is the background noise. $V_{r}$ is the position of the received $r$ and $V_{\mathrm{i}}$ is the vehicle $i$.

The propagation model:

$$
\ell(\mathrm{x}-\mathrm{y})=|\mathrm{x}-\mathrm{y}|^{\alpha}
$$

Where $|x-y|$ denotes the distance between point $x$ of the vehicle and the signal source at point $y$ and the path-loss exponent $\alpha>2$.

So that any received packet less than a specified threshold will be dropped in physical layer.

Network Architecture. This section includes three parts: RSU Capacity, road architecture and RSU layout. Of course, the structure in VANET is still an open answer. But we design the architecture by taking advantages of the assumptions and models above.

In real life, RSUs' abilities are limited by power, computation and other limited resources. The capacity of different RSUs differs, too. So it's necessary to compute the capacity of different traffic scenes and choose the suitable RSU for the road. Capacity is also a key factor in determining the maximum time of a round. So the total number of vehicles under a RSU's coverage is given by us:

$$
\operatorname{Capacity}(\mathrm{n})=\frac{\mathrm{CR}-\frac{\mathrm{WS}}{2}}{\mathrm{VL}+\mathrm{SD}} \times \mathrm{LN} \times \mathrm{n}+\mathrm{LN} \times \mathrm{n}+\frac{\mathrm{WS}}{\mathrm{VL}+\mathrm{SD}} \times \mathrm{LN}
$$

Where CR is the coverage radius of RSU, VL is the average vehicular length, SD is the average safety distance between two adjacent vehicles, $\mathrm{LN}$ is the lane number and $\mathrm{n}$ is the fork number of the intersection.

$\mathrm{WS}=\mathrm{LW} \times \mathrm{LN}+\mathrm{BW} \times \mathrm{BN}$

Where LW is the width of lane, BW is the width of green belt and BN is the number of green belt.

In the motorway scenario, we assume that there are plenty of RSUs along the road. In [22], the author proved that it just needs two orthogonal frequencies to divide the entire road. The RSUs on the left side of the road use a frequency, while the ones on the right use the orthogonal frequency. We use the same road architecture as mentioned:

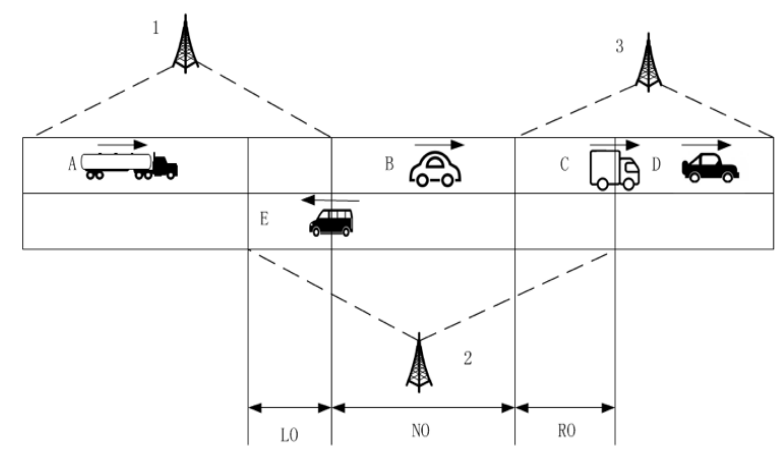

Figure 1. Road architecture

As shown in Fig. 1, the coverage area of a RSU can be divided into three parts: left overlap (LO), no overlap (NO) and right overlap (RO). The cars (Car C and E) in the LO or RO are under the two RSU's management that their frequencies are orthogonal. And that's the reason for the assumption 
of requirement of dual separate antennas. The LO and RO are buffer areas for vehicles to switch from one RSU's service to another's. The algorithm in these areas will be explained later.
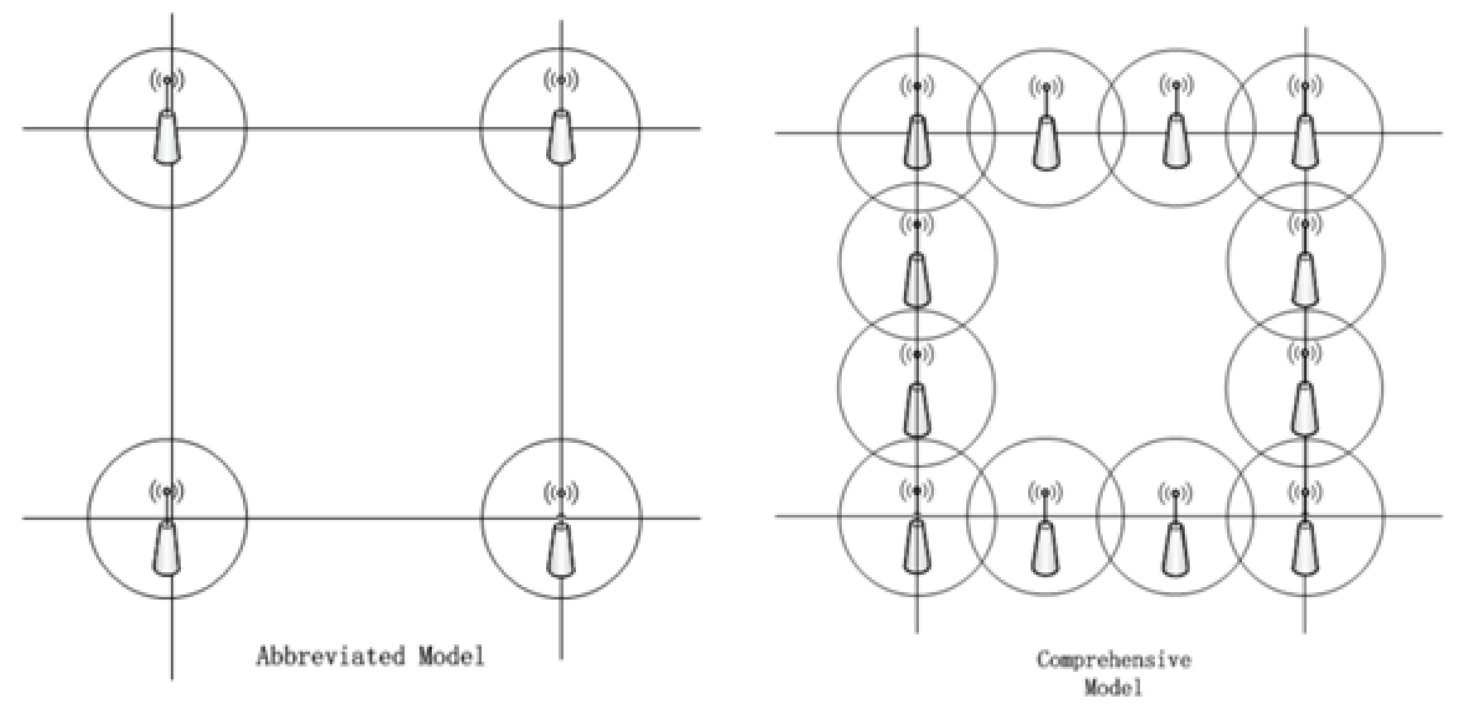

Figure 2. Two layouts

Owing to the cost of erecting RSU, the RSU layout can be divided into two types of comprehensive model and abbreviated model. In comprehensive model, all the roads are under the coverage, which requires considerable number of RSUs. In abbreviated model, equipment is only deployed on the intersections. Due to the saved amounts of RSUs, vehicles are required to switch between DAM and CSMA/CA which may increase the risks. Fig. 2 shows the layouts of two types.

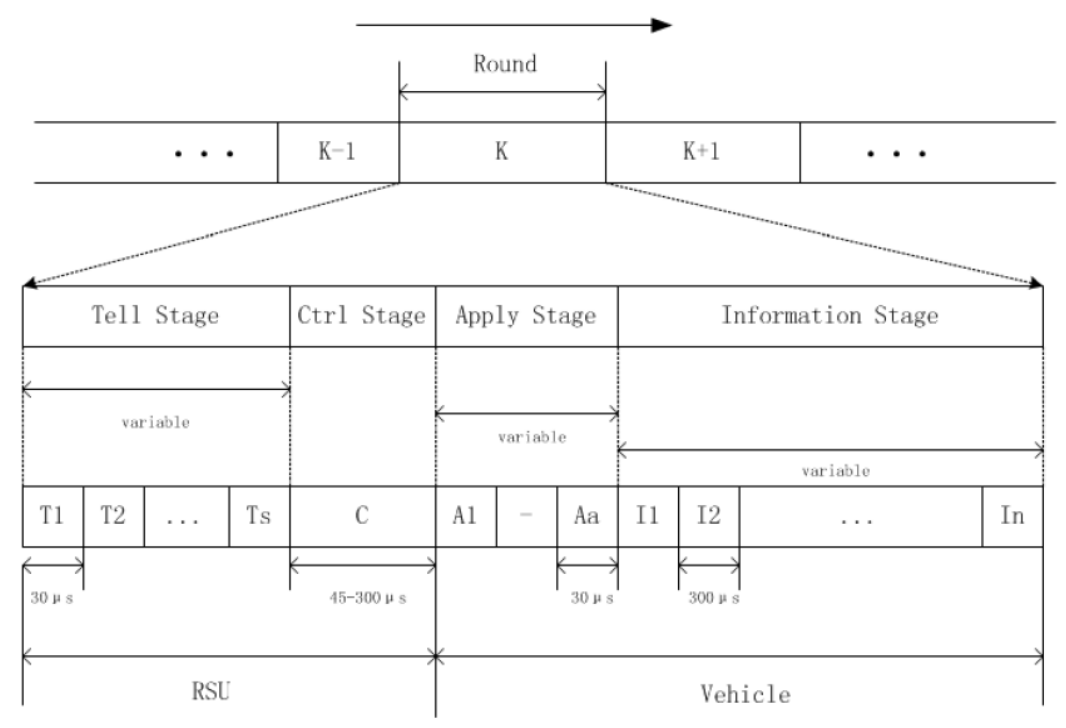

Figure 3. TDMA round structure

TDMA Round Structure and Stages. As illustrated in Fig. 3, time is divided into periods called "round". The duration time of each round could be diverse from each other. Each round has four stages: tell stage, ctrl stage, apply stage and information stage. The ctrl stage is of fixed-length, while the other three stages are of variable-length. The max length of the tell stage depends on the last round's successful applicants. And the length of the apply stage and information stage is under the control of RSU. And the slot time of ctrl stage depends on the RSU's capacity. Thus, the maximum period of one round ( $R_{\max }$ ) is no more than $100 \mathrm{~ms}$, based on that, the concurrent is no greater than two hundred under normal circumstances. 


$$
\mathrm{R}_{\max }=\mathrm{T} \times \text { Capacity }+\mathrm{C}+\mathrm{I} \times \text { Capacity }
$$

Where $\mathrm{T}$ is the slot time of tell stage, $\mathrm{C}$ is the slot time of ctrl stage and $\mathrm{I}$ is the slot time of information stage.

In our DAM protocol, the first two stages are mainly for RSU, while the last two stages for vehicles. The RSU phase is reserved for RSU to send and broadcast, which requires the vehicles to be quiet. In tell stage, the transmitted packet includes a local area id called Mapping Number (MN) which is not universal. And the control packet includes the length of apply stage and a map called Bit Map (BM) indicating the arrangement of time slot in information stage.

Like the RSU phase, the vehicle phase is consisted of two variable stages under the control of RSUs. The apply stage's length is chosen by the policy of RSU, which roughly dominates the numbers of access vehicles. In apply stage, vehicles send apply messages to RSU by employing CSMA/CA. So that the number of a round's successful applications is unidentified, which is decided on a case-by-case basis. Finally, the vehicles occupy the wireless channel in proper order parsed from the MM, while RSU can be dormant.

\section{Details of DAM Protocol}

Packet Format. Tell packets are used for RSU to inform the vehicles which successful request for the MN used in the local domain. RSU adopts broadcast to send the tell packet to each successful requested vehicle. We design the structure of tell packet as shown in Fig. 4.

That in Source ID and Destination ID field is the global unique ID of RSU and Vehicle. The Mapping Number field is the local area number given by RSU. Then the Check Sum field is used for cyclic redundancy check (CRC).

\begin{tabular}{|c|c|}
\hline \multicolumn{2}{|c|}{$\begin{array}{l}\text { Source ID } \\
\text { (32 bits) }\end{array}$} \\
\hline \multicolumn{2}{|c|}{$\begin{array}{l}\text { Destination ID } \\
\quad(32 \text { bits })\end{array}$} \\
\hline $\begin{array}{l}\text { Mapping Number } \\
\quad(16 \text { bits })\end{array}$ & $\begin{array}{l}\text { Check Sum } \\
\text { (16 bits) }\end{array}$ \\
\hline
\end{tabular}

Figure 4. Tell packet format

Ctrl packets are used for RSU to broadcast controlling information serve for the next coming two stages. The RSU makes the BM according to the usage state of the MNM and fill it in the packet. The RSU should figure out the length of apply stage and fill in the packet on the basis of capacity, Mapping Number Map and RSU's policy. What's more, the RSU should choose the appropriate algorithm used in apply stage. We design the structure of ctrl packet as shown in Fig. 5.

\begin{tabular}{|c|c|}
\hline \multicolumn{2}{|c|}{$\begin{array}{l}\text { Sequence } \\
\text { (32 bits) }\end{array}$} \\
\hline \multicolumn{2}{|c|}{$\begin{array}{l}\text { Source ID } \\
\text { (32 bits) }\end{array}$} \\
\hline \multicolumn{2}{|c|}{$\begin{array}{c}\text { Mapping Map } \\
\text { (Variable) } \\
{[32-1024 \text { bits depends on Capacity }]}\end{array}$} \\
\hline $\begin{array}{l}\text { Algorithm } \\
\text { (16 bits) }\end{array}$ & $\begin{array}{c}\text { Length } \\
\text { (16 bits) }\end{array}$ \\
\hline & \\
\hline
\end{tabular}

Figure 5. Ctrl packet format 
That in Sequence field is the sequence of the ctrl packet. The Source ID field is the global unique ID of RSU. The size of Mapping Map field is determined by the capacity of the RSU, which is required to be multiple of 4 bits. And the size of MM is immutable in specific RSU. The algorithm field is the short description of algorithm used in the apply stage. The length field is the key parameter for apply stage. Then, the Check Sum field is for CRC.

Apply packets are used for vehicles to request for the permission of the time slot in information stage. Vehicles choose a random number from zero to the length minus one resolving from the ctrl packet. We design the structure of apply packet as shown in Fig. 6.

\begin{tabular}{|c|c|}
\hline \multicolumn{2}{|c|}{$\begin{array}{c}\text { Source ID } \\
(32 \text { bits })\end{array}$} \\
\hline \multicolumn{2}{|c|}{$\begin{array}{c}\text { Destination ID } \\
(32 \text { bits })\end{array}$} \\
\hline Rounds & Check Sum \\
$(16$ bits $)$ & $(16$ bits $)$ \\
\hline
\end{tabular}

Figure 6. Apply packet format

That in Source ID and Destination ID field is the global unique ID of Vehicle and RSU. The Rounds field is the number of the rounds which vehicles request for. And the Check Sum field is for CRC.

Info packets are used for vehicles to send security information. We design the structure of info packet as shown in Fig. 7.

\begin{tabular}{|c|}
\hline $\begin{array}{c}\text { Sequence } \\
(32 \text { bits })\end{array}$ \\
\hline $\begin{array}{c}\text { Source ID } \\
(32 \text { bits })\end{array}$ \\
\hline $\begin{array}{c}\text { Destination ID } \\
(32 \text { bits })\end{array}$ \\
\hline $\begin{array}{c}\text { Forward ID } \\
(32 \text { bits })\end{array}$ \\
\\
\\
Security Message \\
$(1024$ bits $)$ \\
\\
\hline $\begin{array}{c}\text { Check Sum } \\
(32 \text { bits })\end{array}$ \\
\hline
\end{tabular}

Figure 7. Info packet format

That in Sequence field is the sequence of the info packet. The Source ID, Destination ID and Forward ID are all global unique IDs of source vehicle, destination and relay node. The Security Message field is the key message for VANET Safety Services. The last Check Sum field is for CRC.

RSU Map Specification. In our protocol, there are two kind of maps used by RSUs: Mapping Number Map (MNM) and Bit Map(BM). MNM is for RSU to remember the usage of local number. When an apply packet arrives, the RSU queries for the available mapping number in MNM. Once the counter of vehicular demand goes to zero, RSU removes the information in MNM and revokes the given out $\mathrm{MN}$.

Bit Map is a particular time slot assignment schedule of information stage. Vehicles get their sending order by resolving the BM. BM is made by RSU in the light of capacity and vehicular demand. The size of BM is the least multiple of 4 bits and meets the capacity.

RSU Workflow. RSU must work on the first three stages in one round, and could hibernate 
during the last stage. In the tell stage, RSU sends tell packets to each successful applied vehicle last round. After that, the RSU broadcasts the produced BM, the apply stage's size and the back off algorithm chosen based on the circumstances. After being speaker in the first two stages, RSU becomes a listener or a forwarder in the next two stages. In apply stage, RSU listen to the media to collect the apply information from vehicles. It can occur that RSU gathers no request during the stage, which will lead to the absence of the tell stage in the next round. Of course, if the number of vehicles reaches the capacity of the RSU, the apply stage would jump over according to the policy. So, to be exact, a round consists of two to four stages, that means the tell stage and apply stage could be skipped. But, the ctrl stage and information stage are integral parts of a round. In the last stage of the round, the RSU could hibernate to save energy. And it can be a forwarder to make a contribution to the safety information propagation.

In our DAM protocol, the RSU will expand or shrink (even skip) some variable stages according to the number of vehicles which have the requirements to send message. The length of a round is uncertain, but it has ceilings based on the RSU's capacity. If the requirements from vehicles get more, the RSU will increase the length of information stage and decrease the length of apply stage to a certain extent. In contrast, the RSU would decrease the length of information stage and keep or increase the length of apply stage. And the state of addition or reduction depends on the number of successfully applied vehicles, whose upper bound is last apply stage's length. In our protocol, all vehicles are served equal so that it can realize adaptability and fairness.

The details of the algorithm for RSU workflow are shown in Table 1.

Table 1 RSU workflow algorithm

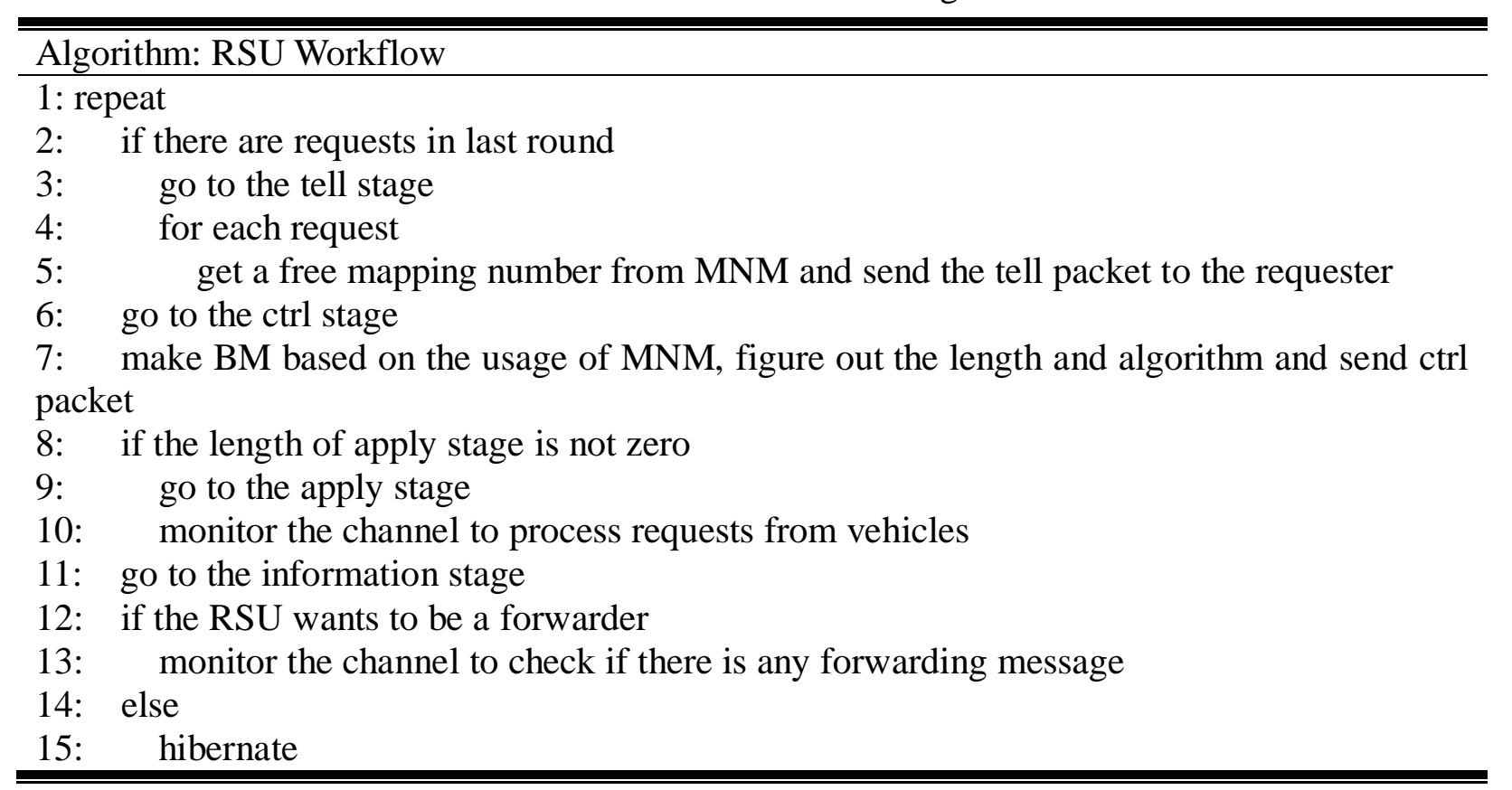

Vehicle Workflow. New vehicle participates in:

A vehicle which has the demand to send safety messages must listen to the wireless channel until getting the control information while in the scope of management of RSU. The waiting time will last less than the maximum time of a round. Generally, the speed of the vehicle is no more than $50 \mathrm{~m} / \mathrm{s}$ in the most cases, that the moving distance during the time is no greater than 5 meters which is acceptable. After resolving the control information, the vehicle will know when the apply stage starts and how long the stage will be sustained. Then it can choose the network wants in.

A vehicle which wants to participate in the network has the following two cases: For the vehicle $\mathrm{C}$ and vehicle $\mathrm{E}$ in Fig. 1, the vehicles are both in the overlap area where have two orthogonal frequencies. If the period in the remaining overlap could meet the demand of the vehicle, there are three options: the short service network (RSU 2), the long service network (RSU 3 for vehicle C 
and RSU 1 for vehicle E) and both. For the vehicle B in Fig. 1, it's in none overlap area of RSU 2. It can only participate in the only option.

The details of the algorithm for new vehicle to participate in are shown in Table 2.

Table 2 Algorithm for new vehicle participates in

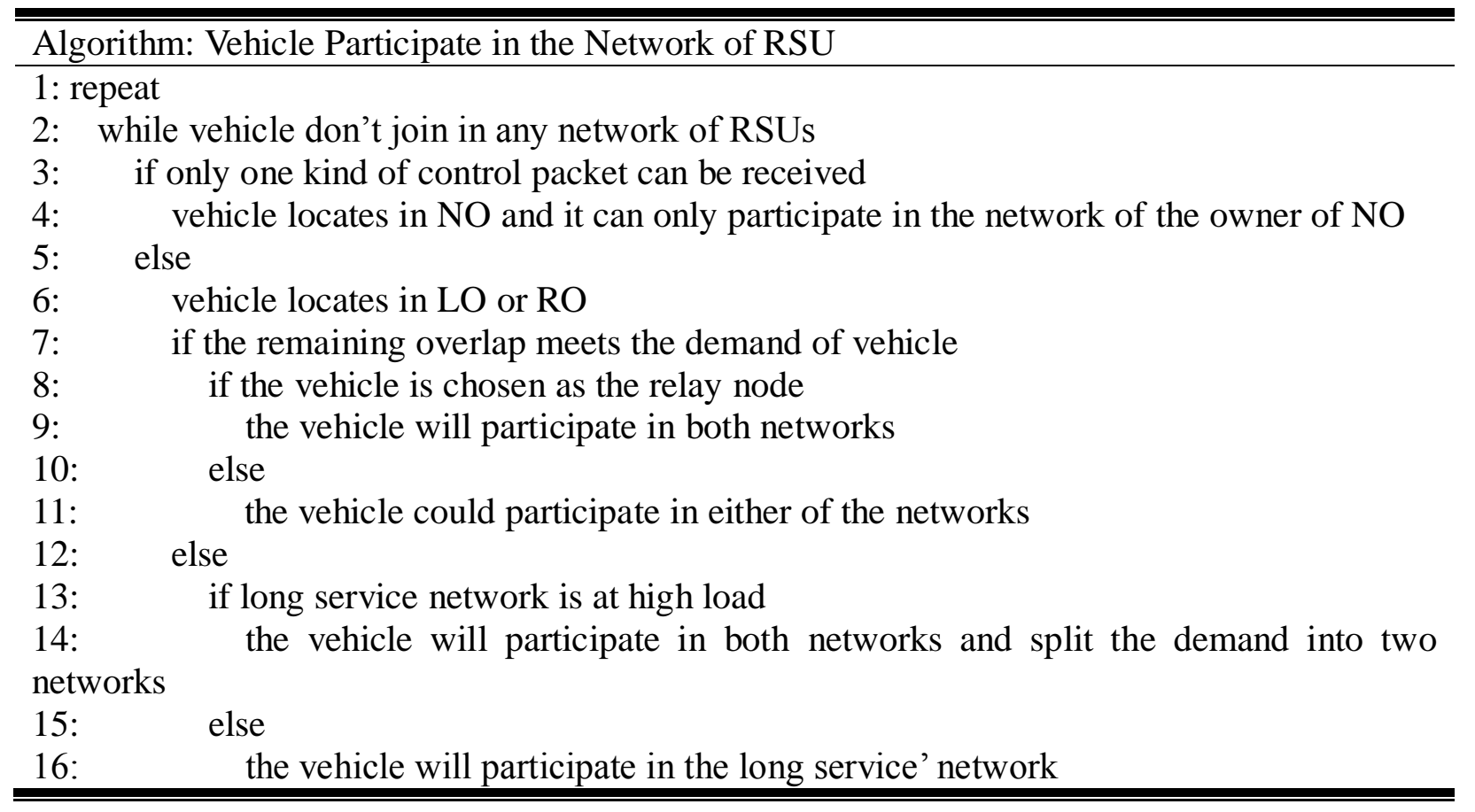

After choosing the wanted-in network, the vehicle could use the current algorithm to apply for the time slot from the network's master. Once the vehicle gets the authorization from the next round's control information, it can send safety messages without collision in the round.

Old vehicle drops out:

Owing to the Rounds field in apply packet, the vehicle which meets the demand just drops out of the control of RSU. After each round, the RSU will maintain its MNM and minus the round number of each vehicle. Once the number down to zero, the given out number will be revoked.

Vehicle workflow algorithm:

The details of the algorithm for vehicle in are shown in Table 3.

Table 3 Vehicle workflow algorithm

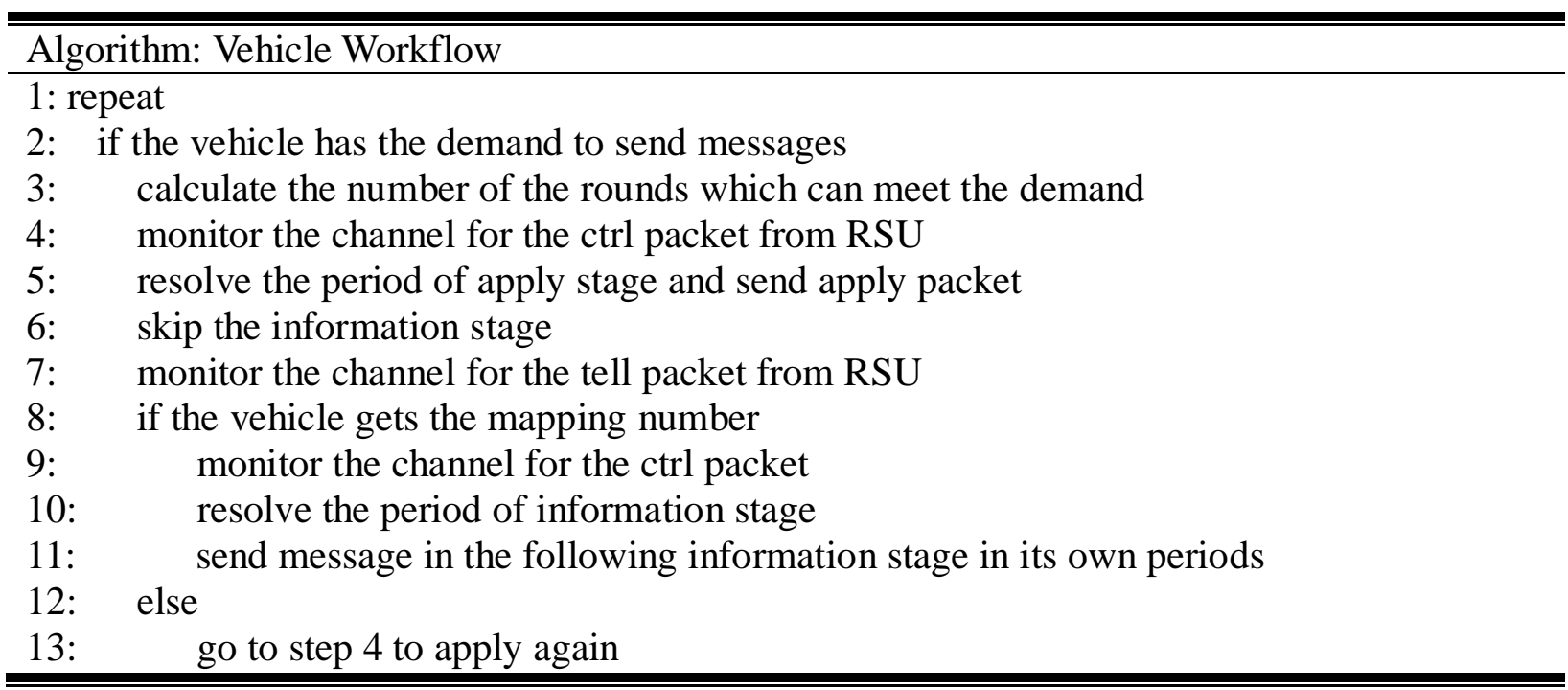


Specialization in the Apply Stage. In this part, we will show the specialization in the apply stage. As is illustrated above, the vehicles adopt CSMA/CA without ACK and RTS/CTS in apply stage. The superiority of this can be seen in the following example.

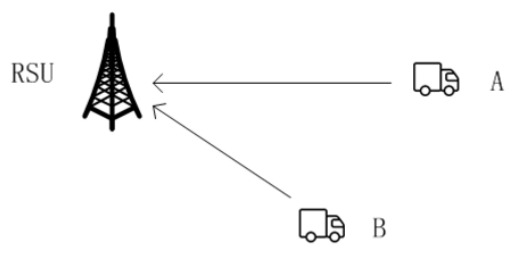

Figure 8. Superiority of CSMA/CA without ACK and RTS/CTS

As shown in Fig. 8, vehicle A and B send the apply packet to the RSU at the same time, while the distance between RSU and B is shorter than the distance between RSU and A. In the normal CSMA/CA used in IEEE 802.11p, this means the packet collision occurred. But by adopting the CSMA/CA without ACK and RTS/CTS, the RSU can successful accept the request from B because that the B's packet arrives before A. So this point can greatly reduce the influences from collisions caused by more than one terminal randomly choosing the same number.

\section{Performance Evaluation}

Simulation Environment. We had implemented our DAM protocol and modified 802.11p on the OPNET Modeler and used the simulation results of ACFM protocol from [22]. In our simulation scenario, a $500 \mathrm{~m} \times 500 \mathrm{~m}$ square road network is created. Each vehicle broadcast an information packet of 1024 bytes every 100ms generally used in VANET safety applications. There are from 20 to 200 vehicles in the simulation, and the simulation time is set to $1000 \mathrm{~s}$ in each case. The data transmission rate is $4 \mathrm{Mbps}$ in the simulation. And about the length of apply stage, we simply let the length be twice as much as capacity minus existing vehicles number.

Simulation Results. Fig. 9 shows the average end-to-end delay of the three protocols in different subnet density scenarios. 802.11p has the lower delay at first, but it will grow logarithmically along with the increase of the subnet density. The ACFM has the delay less than 100ms in most scenarios. But the delay of our DAM protocol is less than $50 \%$ of ACFM.

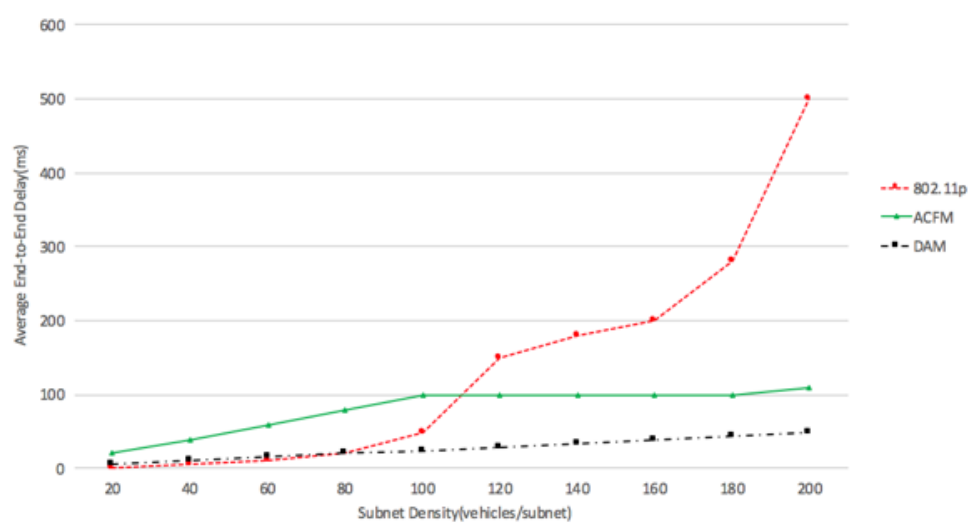

Figure 9. Performance: average end-to-end delay

Fig. 10 shows the packet loss ratio of the three protocols. The packet loss ratio rises along with the increase of subnet density in other two protocols, but falls in our protocol. And our DAM protocol has the lowest packet loss ratio in the majority of cases thanks to the simplex apply. 


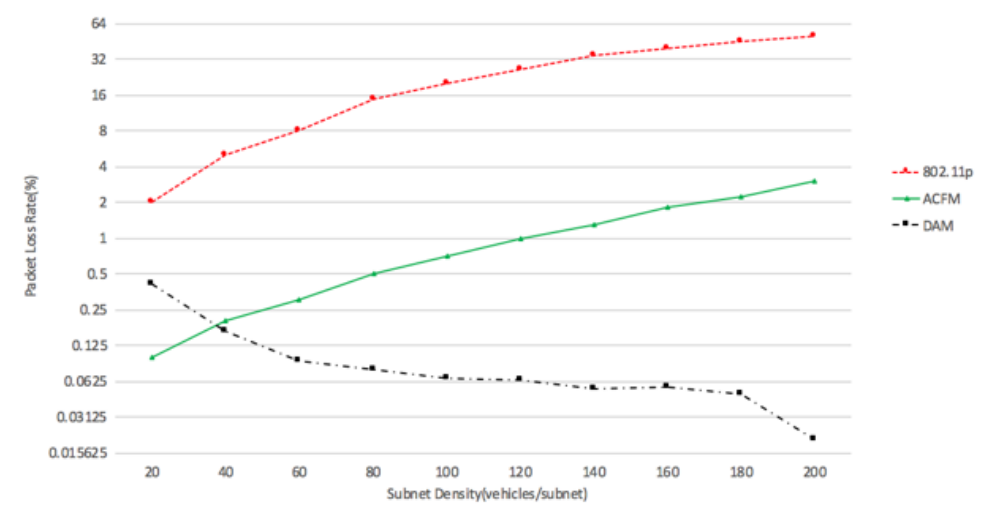

Figure 10. Performance: packet loss ratio

Fig. 11 shows the information packet loss ratio. In 802.11 , there is only information packet. In ACFM, there are control packet from RSU and the information packet. But the packet loss is only due to the information packet. So the information packet loss ratio is the same as packet loss ratio in 802.11p and ACFM. But in our DAM protocol, the information packet loss ratio will always be zero.

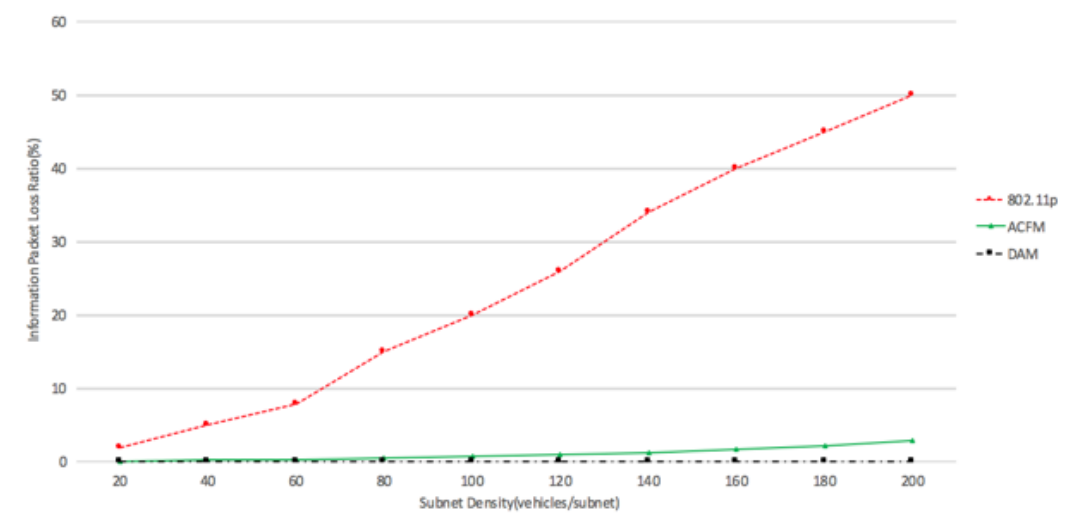

Figure 11. Performance: information packet loss ratio

\section{Conclusion}

In this paper, we propose a new demand-adaptive MAC protocol without information packet collision for VANET Safety Services. In our DAM protocol, the RSU and the vehicles within the scope of its management form an independent network. The RSU, as the dominant of the system, is supposed to process requests, allocate time slots and broadcast messages. And the vehicles need to perform under the control. By shrinking or expanding the slots and playing the natural advantage of TDMA, DAM protocol can realize both the self-adaptive demand and free collision of information packet. Our scheme has advantages in not only average end-to-end delay but also packet loss rate compared to the modified IEEE 802.11p standard and ACFM protocol.

\section{References}

[1] European Commission: Annual Accident Report 2015, Jul. 2015.

[2] R. F. Rozycki: Persons Fatally Injured in Motor Vehicle Crashes, 1980 - 2014 (1), Feb. 2016.

[3] National Bureau of Statistics: 2015 National Economic and Social Development Statistics Bulletin, Feb. 2016. (In Chinese)

[4] M. Bayly, M. Regan and S. Hosking: Intelligent transport systems and motorcycle safety (Monash University Accident Research Centre, Australia, 2007), p. 1-9. 
[5] T. Liu, Z. Li, X. Xia and S. Luo: 2009 5th International Conference on Wireless Communications, Networking and Mobile Computing (Beijing, China, Sep. 24-26, 2009), p. 1-4.

[6] H. Mahmood, C. Comaniciu: 2005 International Conference on Collaborative Computing: Networking, Applications and Worksharing (San Jose, CA, USA, Dec. 19-22, 2005), p. 1-7.

[7] D. Jiang, V. Taliwal, A. Meier, W. Holfelder and R. Herrtwich: IEEE Wireless Communications, Vol. 13 (2006) No.5, p.36-43.

[8] A. Dua, N. Kumar, S. Bawa, and N. Chilamkurti: Wireless and Pervasive Computing (ISWPC), 2013 International Symposium on (Taipei, China, Nov. 20-22, 2013), p. 1-6.

[9] D. Verenzuela, C. Liu, L. Wang, and L. Shi: 2014 IEEE 80th Vehicular Technology Conference (Vancouver, BC, Canada, Sep. 14-17, 2014), p. 1-5.

[10]H. Cozzetti, R. Scopigno, L. Casone and G. Barba: Services Computing Conference, 2009. APSCC 2009. IEEE Asia-Pacific (Singapore, Dec. 7-11, 2009), p. 1-6.

[11]J. Blum and A. Eskandarian: IEEE Transactions on Intelligent Transportation Systems, Vol. 8 (2007) No.1, p. 4-13.

[12]Z. Doukha and S. Moussaoui: IEEE Transactions on Vehicular Technology, Vol. 65 (2015) No.2, p. 603-613.

[13]H. Menouar, F. Filali and M. Lenardi: IEEE Wireless Communications, Vol. 13 (2006) No.5, p. $1-6$,

[14]G. Korkmaz, E. Ekici, F. Özgüner and Ü Özgüner: Vanet '04 Vehicular Ad-hoc Networks Workshop (Philadelphia, PA, USA, Oct. 01-01, 2004), p. 1-10.

[15]G. Korkmaz, E. Ekici, F. Özgüner and Ü Özgüner: 2006 IEEE International Conference on Communications (Istanbul, Turkey, Jun. 11-15, 2006), p. 1-6.

[16] V. Bharghavan, A. Demers, S. Shenker and L. Zhang: COMM94 ACM SIGCOMM '94 (London, England, UK, Aug. 31-Sep. 02, 1994), p. 212-225.

[17]J. Deng, P. Varshney and Z. Haas: A new backoff algorithm for the IEEE 802.11 distributed coordination function (Cornell University, USA, 2004), p. 1-6.

[18]N.O. Song, B.J. Kwak, J. Song and L.E. Miller: Vehicular Technology Conference, 2003. VTC 2003-Spring. The 57th IEEE Semiannual (Jeju, South Korea, Apr. 22-25, 2003), Vol. 4, p. 1-4.

[19]Q. Nasir and M. Albalt: Communication Networks and Services Research Conference, 2008. CNSR 2008. 6th Annual (Halifax, NS, Canada, May 5-8, 2008), p. 533-538.

[20]R. Zhang, X. Cheng, L. Yang, X. Shen and B. Jiao: IEEE Transactions on Intelligent Transportation Systems, Vol. 16 (2014) No.1, p. 411-416.

[21]X. Jiang and D. Du: IEEE Transactions on Vehicular Technology, Vol. PP (2016) No.99, p. 1-1.

[22]W. Guo, L. Huang, L. Chen, H. Xu and J. Xie: Wireless Communications \& Signal Processing (WCSP), 2012 International Conference on (Huangshan, China, Oct. 25-27, 2012), p. 1-6.

[23]ETST: Intelligent Transport Systems (ITS); Access layer specification for Intelligent Transport Systems operating in the $5 \mathrm{GHz}$ frequency band, p. 1-48, Nov. 2012. 